\title{
A new very faint $X$-ray transient in the Galactic center
}

\author{
Simona Soldi ${ }^{1}$, Maïca Clavel ${ }^{1,2}$, Andrea Goldwurm ${ }^{1,2}$, \\ Gabriele Ponti ${ }^{3}$, Régis Terrier ${ }^{1}$, Guillaume Trap ${ }^{1,2,4}$, Jochen Greiner ${ }^{3}$, \\ Tobias Prinz ${ }^{3}$, Arne Rau ${ }^{3}$ and Mathieu Servillat ${ }^{2}$ \\ ${ }^{1}$ AstroParticule et Cosmologie, Univ. Paris Diderot, CNRS/IN2P3, CEA/Irfu, Observatoire de \\ Paris, Sorbonne Paris Cité, 10 rue A. Domon et L. Duquet, 75205 Paris Cedex 13, France \\ email: soldi@apc.univ-paris7.fr \\ ${ }^{2}$ Service d'Astrophysique/IRFU/DSM, CEA Saclay, 91191 Gif-sur-Yvette Cedex, France \\ ${ }^{3}$ Max-Planck-Institut für extraterrestrische Physik, D-85748, Garching bei München, Germany \\ ${ }^{4}$ Unité de physique, Palais de la découverte - Universcience, 75008 Paris, France
}

\begin{abstract}
A new X-ray transient, XMMU J174505.3-291445, has been detected within the 2012 XMM-Newton scan of the Galactic center. The short 4.7 ks flare, the highly absorbed Xray spectrum and the relatively low luminosity of the event suggest the association of the source with either the class of very faint X-ray transients or that of supergiant fast X-ray transients. Further analysis, together with the identification of the possible infrared counterpart will help to unveil the true nature of XMMU J174505.3-291445.
\end{abstract}

Keywords. stars: flare, X-rays: binaries, accretion, Galaxy: center

\section{X-ray timing and spectral properties of XMMU J174505.3-291445}

Within the XMM-Newton scan of the Galactic center, a new X-ray transient, XMMU J174505.3-291445, has been detected at about $16^{\prime}$ from $\mathrm{Sgr}^{*}$ (R. A. (J2000) $=$ 17:45:05.3, Dec. $=-29: 14: 46 ; 0.8^{\prime \prime}$ uncertainty at $90 \%$ confidence level), during a flare taking place on 2012 August 31. The source lies within the $2 \sigma$ error circle of 2XMMi J174505.1-291445, a still unidentified object detected on 2006 February 27 and reported in the Third XMM-Newton Serendipitous Source Catalog by Watson et al. (2009).

The 2012 flare of XMMU J174505.3-291445 lasted about $4.7 \mathrm{ks}$, with an average flux of $F(0.2-12 \mathrm{keV})=1.2 \times 10^{-11} \mathrm{erg} \mathrm{cm}^{-2} \mathrm{~s}^{-1}$, corresponding to a luminosity of $\mathrm{L} \sim 10^{35} \mathrm{erg} \mathrm{s}^{-1}$ when assuming a distance of $8 \mathrm{kpc}$ (Figure 1, left panel). About 6 hours after the flare, the luminosity had decreased to $\mathrm{L} \sim 2 \times 10^{33} \mathrm{erg} \mathrm{s}^{-1}$, and the source was not detected in any of the other 2012 scan observations. During the 2006 observation, 2XMMi J174505.1-291445 was detected at an intermediate activity level of L $~ 8 \times$ $10^{33} \mathrm{erg} \mathrm{s}^{-1}$. The combined EPIC MOS+PN spectra obtained during the 2012 flare can be equally well fitted by a power-law model with photon index $\Gamma=2.1 \pm 0.2$ (Figure 1 , right panel) or by a black body with temperature $k T=1.8 \pm 0.1 \mathrm{keV}$, in both cases absorbed by $N_{\mathrm{H}} \sim 20-30 \times 10^{22} \mathrm{~cm}^{-2}$ (all quoted errors correspond to the $90 \%$ confidence level). The quiescence spectrum is best fitted by a black body with $k T=1.1 \pm 0.5 \mathrm{keV}$, absorbed by $N_{\mathrm{H}}=20_{-12}^{+23} \times 10^{22} \mathrm{~cm}^{-2}$. No spectral evolution is observed during the outburst. 

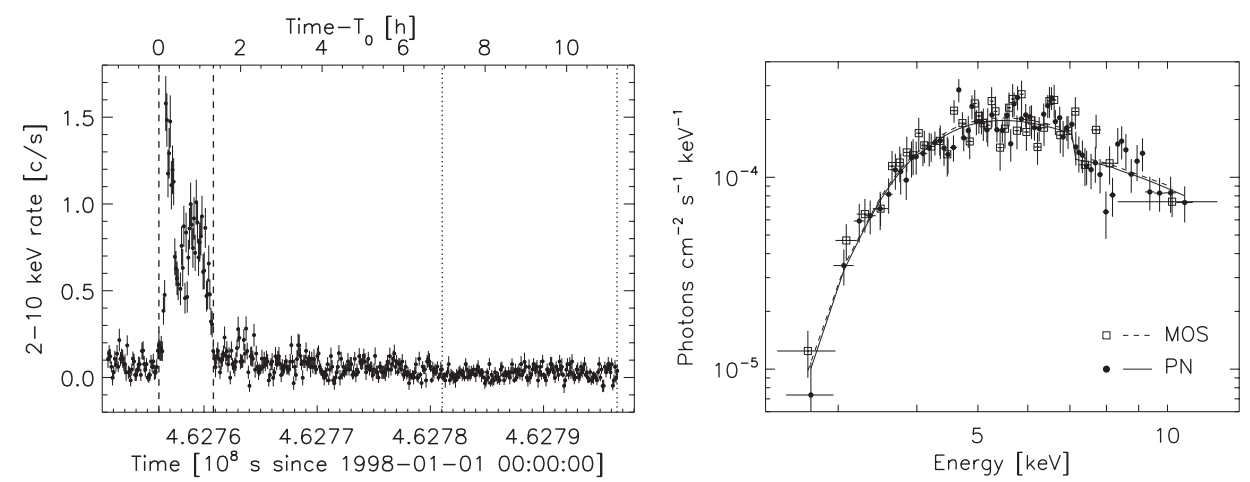

Figure 1. Left: $2-10 \mathrm{keV}$ light curve of XMMU J174505.3-291445 measured by XMM-Newton EPIC/PN on 2012 August 31. The dashed lines mark the flare of the source, while the dotted lines define the quiescence period. Right: EPIC/PN (circles) and EPIC/MOS (squares) spectra during the flare, fitted with an absorbed power-law with $N_{\mathrm{H}}=(27 \pm 2) \times 10^{22} \mathrm{~cm}^{-2}$ and $\Gamma=2.1 \pm 0.2$ (continuous and dashed lines for PN and MOS, respectively).

\section{A new very faint $X$-ray transient?}

The timing and spectral properties of XMMU J174505.3-291445 indicate that it is a distant Galactic source, consistent with a localization within the Galactic center. In particular, the highly absorbed X-ray spectrum excludes the identification with a foreground flaring star. Normal outbursts from bright X-ray binaries generally reach higher luminosities $\left(\mathrm{L}_{\mathrm{X}} \sim 10^{37-39} \mathrm{erg} \mathrm{s}^{-1}\right.$; Chen et al. 1997) and last longer, with the exception of supergiant fast X-ray transient sources, characterized by flares with a duration of a few hours. However, during outbursts these sources normally reach higher luminosities $\left(\mathrm{L}_{\mathrm{X}} \sim\right.$ $10^{36-37} \mathrm{erg} \mathrm{s}^{-1}$ ) and present harder spectra than observed for XMMU J174505.3-291445. On the other hand, the luminosity range of the new transient and its flaring spectrum fit the characteristics of very faint X-ray transients (VFXT), i.e. Galactic X-ray transients with moderate peak X-ray luminosities of $\mathrm{L}_{\mathrm{X}} \sim 10^{34-36} \mathrm{erg} \mathrm{s}^{-1}$ and with quiescent luminosities at, or below, $\mathrm{L}_{\mathrm{X}} \sim 10^{33} \mathrm{erg} \mathrm{s}^{-1}$ (Degenaar \& Wijnands 2009). The origin of such a peculiar activity is still debated, and could be produced by a compact object accreting at a very low rate from the wind of a main sequence companion or in a lowmass X-ray binary with a tight orbit or an unusual donor star (King \& Wijnands 2006, Degenaar et al. 2012). Infrared follow-up observations with the GROND instrument at the MPI/ESO 2.2m telescope on La Silla and with the $6.5 \mathrm{~m}$ Magellan telescope in Las Campanas have been carried out and their analysis is ongoing (Soldi et al. in preparation). The identification of the infrared counterpart will be fundamental to determine the nature of XMMU J174505.3-291445.

\section{Acknowledgements}

This work is partly supported by the UnivEarthS Labex program of Sorbonne Paris Cité (ANR-10-LABX-0023 and ANR-11-IDEX-0005-02). SS and MS acknowledge the Centre National d'Etudes Spatiales (CNES) for financial support. GP acknowledges support via an EU Marie Curie Intra-European fellowship under contract no. FP-PEOPLE2012-IEF-331095. 


\section{References}

Chen, W., Shrader, C. R., \& Livio, M. 1997, ApJ 491, 312

Degenaar, N. \& Wijnands, R. 2009, A\&A 495, 547

Degenaar, N., Wijnands, R., Cackett, E. M., et al. 2012, A $\& A$ 545, A49

King, A. R. \& Wijnands, R. 2006, MNRAS 366, L31

Romano, P. \& Sidoli, L. 2010, Mem. S. A. It. 81, 332

Watson, M. G., Schröder, A. C., Fyfe, D., et al. 2009, A\& A 493, 339 